\title{
Applications and benefits of using gradient percentage depth dose instead of percentage depth dose for electron and photon beams in radiotherapy
}

\author{
Labinot KASTRATI ${ }^{a, b}$, Gezim HOdOLLI ${ }^{a, b,}$, , Sehad KADIRI ${ }^{b, c}$, Elvin DEMIREL ${ }^{d}$, Lutfi ISTREFI ${ }^{e}$, Yllka KABASHI ${ }^{b}$, Burim \\ UKA ${ }^{a, b}$ \\ ${ }^{a}$ University for Business and Technology UBT, Pristina, Kosovo \\ ${ }^{b}$ Institute of Oncology, University Clinical Center of Kosovo, Pristina, Kosovo \\ ${ }^{c} A A B$ College, Pristina, Kosovo \\ ${ }^{d}$ Okmeydan Egitim Hastanesi, Istanbul, Turkey \\ ${ }^{e}$ Faculty of Mathematical Natural Science, University of Pristina, Pristina, Kosovo \\ *E-mail address: g.hodolli@hotmail.com,gezim.hodolli@ubt-uni.net
}

\begin{abstract}
Introduction: The aim of this study is to analyze the gradient of percentage depth dose for photon and electron beams of LINACs and to simplify the data set.

Materials and Methods: Dosimetry measurements were performed in accordance with Technical Reports Series No. 398 IAEA.

Results and discussion: The gradient of percentage depth dose was calculated and compared with the available published data.

Conclusion: Instead of percentage depth dose for increasing and decreasing parts, the findings suggest using only two numbers for specific gradient of dose, separately. In this way, they can replace the whole set of the percentage depth dose (PDD).
\end{abstract}

Key words: beam; energy gradient; linear accelerator.

\section{Introduction}

The medical linear accelerator (LINAC) should pass all procedures of pre-commissioning in order to be used for patient treatment. To validate the treatment planning system (TPS), dosimetry measurements must be performed. Therefore, the minimum data set of percentage depth dose (PDD) and dose profiles (DP) should be gathered for different nominal beam energies, field dimensions, depth, and wedges. For electron beams, wedges cannot be used and instead of field dimensions, the different types of applicators should be used.

Studies have shown that the dose distribution depends on many variables such as types of radiation, the density of the medium, beam energy, source to surface distance (SSD), and field size. ${ }^{1}$ The tolerated limit should be applied throughout the whole process, from planning to delivery dose, including measurements of absorbed dose, calculation, imaging, contouring, and patient setup. According to the international guideline ICRU Report 50, for modality technique threedimensional conformal radiotherapy (3DCRT), the target volume dose variation should be within $+7 \%$ and $-5 \%$ of the value prescribed at the reference point in the tumor volume with the determined absorbed dose value. ${ }^{2}$

For superficial and orthovoltage devices with energy up to $400 \mathrm{kVp}$, the maximum dose for PDD is closer to the surface of the body in comparison to higher $\mathrm{X}$-ray energies, when the maximum dose of the PDD is deeper. This difference occurs because the high-speed electrons are ejected from the surface and subsequent layers. Those electrons deposit their energy deeper in the body from their site of origin.

A photon beam propagating through a phantom is affected by the inverse square law, the attenuation, and the scattering of the photon beam inside the phantom. ${ }^{3}$ Dose distribution on the central axis of the megavoltage photon beam inside the phantom or patient has two main interest regions. In the first interest region, the dose increases rapidly until the dose reaches the maximum value at a specific depth, then at the second interest region, the dose decreases almost exponentially. The shape of the PDD curves for photon and electron beams appears similar for both mentioned regions, yet not identical. 
Traditionally, PDD is used to quantify the rate of increasing and decreasing dose for both mentioned regions. The aim of this study is to propose using the gradient of percentage depth dose (GD) instead of the PDD. The advantages and disadvantages of the dose gradient function are discussed in the next sections. Also, the GD function is calculated and compared with standardized data published on Report 25 of BJR. $^{4}$

\section{Materials and Methods}

The PDD is the ratio expressed as a percentage of absorbed dose on the central axis of the field at depth $d$ to the absorbed dose at the reference point $d_{0}$. The PDD is given by:

$$
P D D\left(d, d_{0}, S, f, E\right)=\frac{D_{d}}{D_{d_{0}}} \times 100 \%
$$

Several parameters affect the central axis depth dose distributions including $\mathrm{d}, \mathrm{d}_{0}$, field surface $\mathrm{S}$, source-to-surface distance $f$, and radiation beam energy $E$. These parameters are given elsewhere. ${ }^{1}$ In the photons' beam with high energy, the dose in the surface depends on the beam's energy and dimensions of the field. ${ }^{3}$ The zone between the surface and the depth of maximum dose, in the range of MV, is known as a build-up zone. ${ }^{5}$ The surface dose is dependent on the machine, the field size, the source to surface distance, the presence of beam modifiers, and the angle of beam incidence. ${ }^{6-10}$ Previous studies have shown that the surface dose increases with increasing field size. ${ }^{5,11}$

When an irradiation beam passes from point $A$ to another point $\mathrm{B}$ with the distance $d$, the distribution beam dose changes from $D_{1}$ to $D_{2}$, given by $\Delta D=\left(D_{2}-D_{1}\right)$, due to the interaction of the beam itself with the material of the tissue. Hence, the unit of gradient dose is percentage per millimeters and its equation is given by:
$G D=\operatorname{grad} D=\frac{\Delta D}{\Delta d}$

Eq. 2

This function represents the difference in the dose based on the specific depth as a result of the interactions of the beam with tissues. ${ }^{12}$ The gradient of the dose depends on the difference of the dose divided by the difference of the distance for two specific points.

The PDDs for photons and electron beams with $10 \times 10 \mathrm{~cm}^{2}$ for the first LINAC are presented in Figure 1. Rhombuses, triangles, and circles in the same figure represent the data of $10 \times 10 \mathrm{~cm}^{2}$ from Report 25 of $\mathrm{BJR}^{4}$ for photon beams with 6 and $15 \mathrm{MV}$ and electron beam $12 \mathrm{MeV}$, respectively. The data of the build-up region of PDD are not available for comparison. So, it is very important to identify the maximum depth dose, which is a distinguishing point between the two regions.

As such, to compare those parameters, the calculation of the first and the second region of the GD is given by:

$G D^{ \pm}=\frac{D_{2}-D_{1}}{d_{2}-d_{1}}$

Eq. 3

Depending on the regions, there are two options, region 1 and region 2, respectively $\mathrm{GD}^{+}$and $\mathrm{GD}^{-}$. For the GD+ parameters, D1 represents the surface dose and D2 the maximum achieved dose. On the other hand, $d_{1}$ and $d_{2}$ are the specific depths for the respective doses in the first region. So, when $D_{2}>D_{1}$ and $d_{2}>d_{1}$, the GD is positive and belongs to the first region of the curve.

On the other hand, for the $\mathrm{GD}^{-}$parameters, D1 represents the maximum achieved dose, while D2 represents the same values as the surface dose. $d_{1}$ and $d_{2}$ are specific depths for the respective doses in the second region. The entire equation is best visualized in Figure 2. So, when $D_{2}<D_{1}$ and $d_{2}>d_{1}$, the GD is negative and belongs to the second region of the curve.
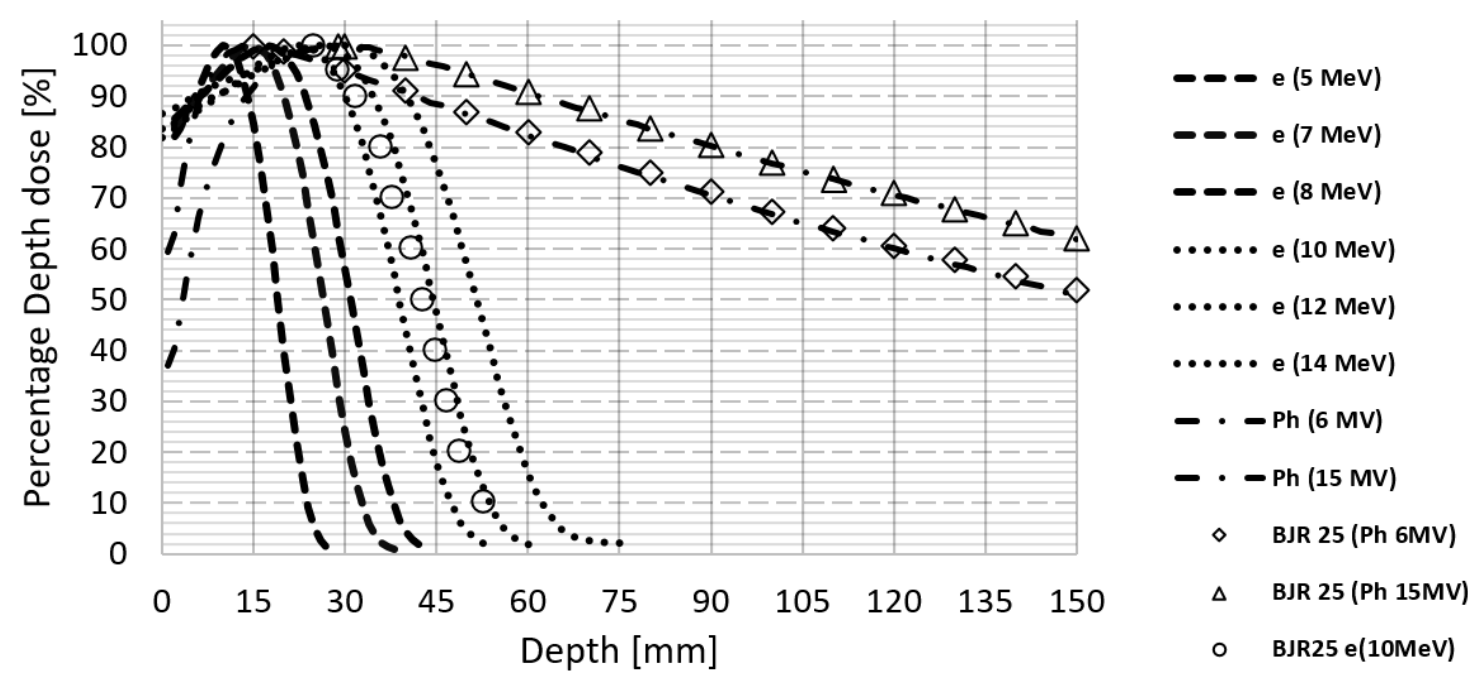

Figure 1. Percentage depth dose for photons and electrons fields $10 \times 10 \mathrm{~cm}^{2}$ 
Therefore, to calculate $\mathrm{GD}^{ \pm}$, the calculation should be done separately for $\mathrm{GD}^{+}$and $\mathrm{GD}^{-}$, the first one belongs to the Iregion and the second one belongs to the II-region. The regions are schematically presented in Figure 2.

Therefore, the idea is to use the $\mathrm{GD}^{-}$function for both interested regions instead of the data set for PDD. The GD function expresses the quantity of changing rate of dose through the central axis.

The irradiation experiment was performed at the Radiotherapy Department of University Clinical Center of Kosovo. The Siemens Primus LINACs were used to produce photon and electron beams with field dimensions $10 \times 10 \mathrm{~cm}^{2}$ at surface skin distance (SSD) $100 \mathrm{~cm}$. The nominal accelerator energy of photon beams was 6 and $15 \mathrm{MV}$ and for the electron beams $5,7,8,10,12$ and $14 \mathrm{MeV}$. With that being said, the modalities and energy modes were the gathered data sets to create the PDDs, which afterwards, were calculated for the GD function.
The absolute dosimetry is done in accordance with IAEA Technical Report Series no $398^{3}$ and is practically described in detail somewhere else. $^{13,14}$

For measuring PDD of LINAC beams a PTW MP3 motorized water phantom system was used with the calibrated semiflex ionization chamber, type 31010. For beam analyzer and controlling measuring system, Mephysto $\mathrm{mc}^{2}$, version 7.4, were used.

\section{Results and Discussion}

In accordance with Equation 2, from the measured PDD of LINAC (Siemens Primus), $\mathrm{GD}^{+}$and $\mathrm{GD}^{-}$were calculated for photon beams with $6 \mathrm{MV}$ and $15 \mathrm{MV}$, with field dimension $10 \times 10 \mathrm{~cm}^{2}$, used for medical purposes at the radiotherapy department. Table 1 presents the input data and calculated results.

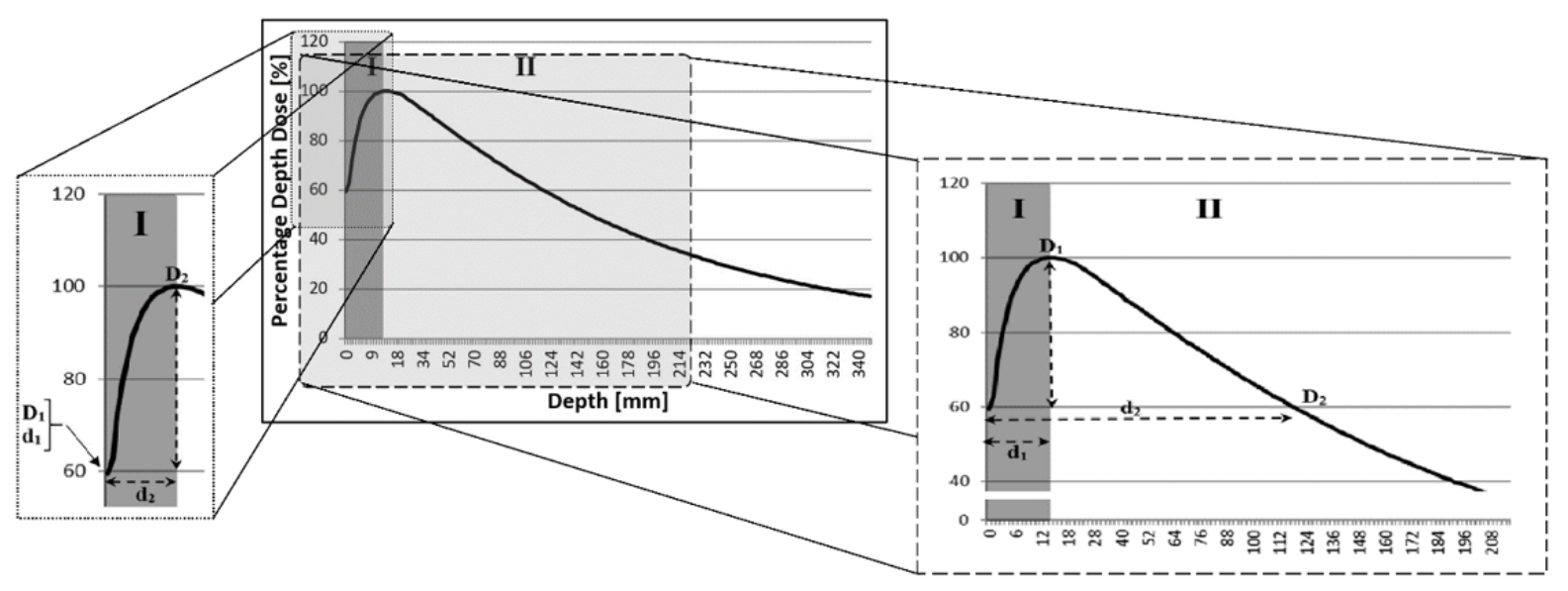

Figure 2. Schematic view of GD+ and GD- for gamma rays

Table 1. Gradient results for photon and electron beams

\begin{tabular}{|c|c|c|c|c|c|c|c|}
\hline $\mathbf{N r}$ & Nominal Energies & GD & $\begin{array}{c}D_{1} \\
{[\%]}\end{array}$ & $\begin{array}{c}\mathrm{D}_{2} \\
{[\%]}\end{array}$ & $\begin{array}{c}d_{1} \\
{[\mathrm{~mm}]}\end{array}$ & $\begin{array}{c}d_{2} \\
{[\mathrm{~mm}]}\end{array}$ & $\begin{array}{l}\text { GD-result } \\
{[\% / \mathrm{mm}]}\end{array}$ \\
\hline 1 & \multirow{2}{*}{$6 \mathrm{MV}$} & $\mathrm{GD}^{+}$ & 59.4 & 100.0 & 1.0 & 14.5 & 2.798 \\
\hline 2 & & $\mathrm{GD}^{-}$ & 100.0 & 59.4 & 14.5 & 122.0 & -0.377 \\
\hline 3 & $6 \mathrm{MV}-\mathrm{BJR} 25$ & $\mathrm{GD}^{-}$ & 100.0 & 59.4 & 15.0 & 124.6 & -0.370 \\
\hline 4 & \multirow{2}{*}{$15 \mathrm{MV}$} & $\mathrm{GD}^{+}$ & 37.0 & 100.0 & 1.0 & 29.0 & 2.171 \\
\hline 5 & & $\mathrm{GD}^{-}$ & 100.0 & 37.0 & 29.0 & 269.0 & -0.262 \\
\hline 6 & $15 \mathrm{MV}-\mathrm{BJR} 25$ & $\mathrm{GD}^{-}$ & 100.0 & 37.0 & 29.0 & 279.0 & -0.252 \\
\hline 7 & \multirow{2}{*}{$5 \mathrm{MeV}$} & GD+ & 82.1 & 100.0 & 1.0 & 10.0 & 1.795 \\
\hline 8 & & GD- & 100.0 & 82.1 & 10.0 & 15.5 & -3.264 \\
\hline 9 & \multirow{2}{*}{$7 \mathrm{MeV}$} & GD+ & 84.2 & 100.0 & 1.0 & 15.0 & 1.053 \\
\hline 10 & & GD- & 100.0 & 84.2 & 15.0 & 21.5 & -2.431 \\
\hline 11 & \multirow{2}{*}{$8 \mathrm{MeV}$} & GD+ & 85.7 & 100.0 & 1.0 & 17.0 & 0.842 \\
\hline 12 & & GD- & 100.0 & 85.7 & 17.0 & 25.0 & -1.790 \\
\hline 13 & \multirow{2}{*}{$10 \mathrm{MeV}$} & GD+ & 81.9 & 100.0 & 1.0 & 23.0 & 0.788 \\
\hline 14 & & GD- & 100.0 & 81.9 & 23.0 & 33.2 & -1.777 \\
\hline 15 & $10 \mathrm{MeV}$ BJR 25 & GD- & 100.0 & 81.9 & 25.0 & 35.0 & -1.810 \\
\hline 16 & \multirow{2}{*}{$12 \mathrm{MeV}$} & GD+ & 83.5 & 100.0 & 1.0 & 26.0 & 0.633 \\
\hline 17 & & GD- & 100.0 & 83.5 & 26.0 & 37.0 & -1.497 \\
\hline 18 & \multirow{2}{*}{$14 \mathrm{MeV}$} & GD+ & 86.5 & 100.0 & 1.0 & 29.0 & 0.467 \\
\hline 19 & & GD- & 100.0 & 86.5 & 29.0 & 41.8 & -1.058 \\
\hline
\end{tabular}




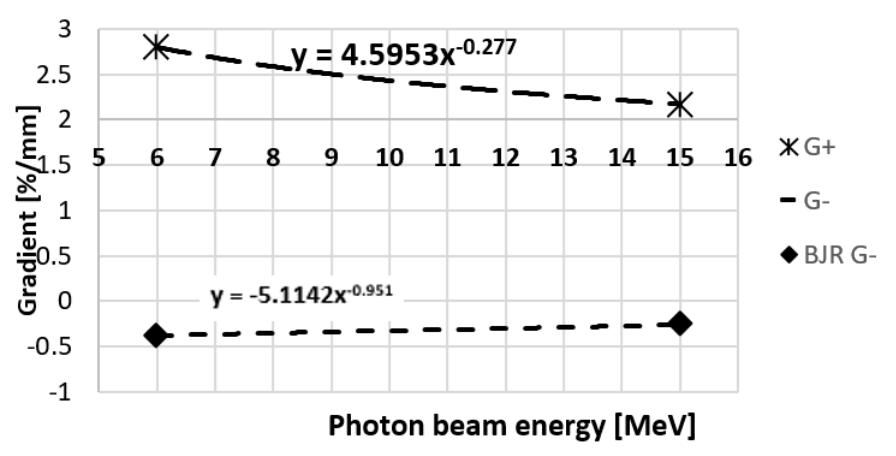

Figure 3. Percentage of Gradient Dose for $6 \mathrm{MV}$ and $15 \mathrm{MV}$

Measuring the surface dose is the most challenging part of the PDD curve. So, it will increase the uncertainty in the determination of the $\mathrm{GD}^{+}$function. However, the quality of the measured dose at the surface is not the aim of this study.

To calculate the $\mathrm{GD}^{+}$, Equation 3 is taken into account. For first region of photon beam with energy $6 \mathrm{MV}$, the input data were: $\mathrm{D}_{2}=100 \%, \mathrm{D}_{1}=59.43 \%, \mathrm{~d}_{2}=14.5 \mathrm{~mm}$ and $\mathrm{d}_{1}=1.0$ $\mathrm{mm}$. The same approach was used for the calculation of the $\mathrm{GD}^{+}$for $15 \mathrm{MV}$ beam.

Equation 3 was used again for the calculation of the GD which represents the second region of the PDD curve with energy $6 \mathrm{MV}$ as shown in Figure 2. For this case, the data were: $\mathrm{D}_{2}=59.43 \%, \mathrm{D}_{1}=100 \%, \mathrm{~d}_{2}=122 \mathrm{~mm}$ and $\mathrm{d}_{1}=14.5$ mm.

Figure 3 presents the calculated values of the $\mathrm{GD}^{+}$and the

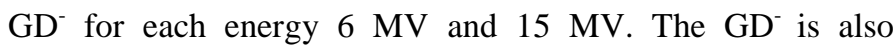
calculated in the same way with the data from Report 25 of the British Journal of Radiology. $\mathrm{GD}^{+}$cannot be calculated due to the lack of data available in BJR.

The $\mathrm{GD}^{-}$calculated values of this research and the $\mathrm{GD}^{-}$of the calculated data from BJR are identical up to the second decimal place.

To find the $\mathrm{GD}^{+}$and the $\mathrm{GD}^{-}$of electron beams, the same procedure is applied as described for photon beams. More data for other nominal energies can be seen in Table 1. Gradients for electron beams with energy 5, 7, 8, 10, 12, and $14 \mathrm{MeV}$ are

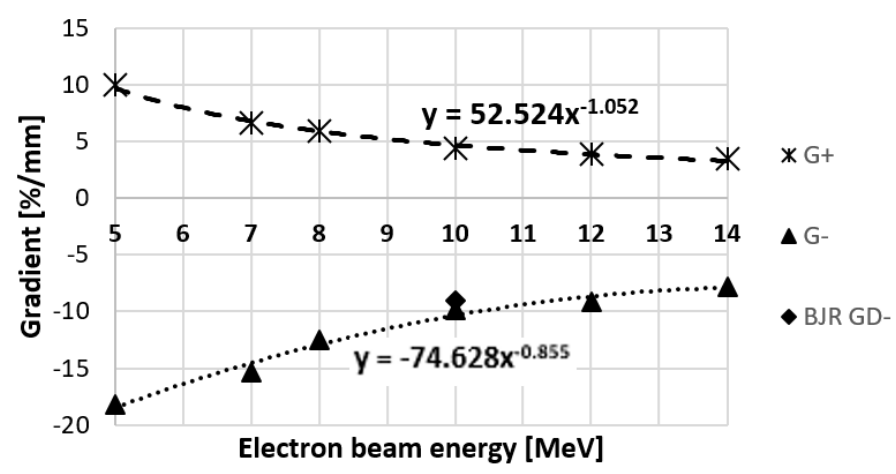

Figure 4. Percentage of Gradient Dose for electron beams

presented in Figure 4. Only $10 \mathrm{MeV}$ data of electron energy beam is available in Report 25 of BJR.

The best fitting for the electron beam energies is exponential, like it is shown in Figure 4. Based on that, the same nature of phenomena should be respected for the photon beam energies. So, for this reason, the exponential fitting is used as well for the photons beams.

\section{Conclusions}

For a PDD, it is necessary to have a set of data with a percentage dose and a specific depth. Therefore, the findings suggest that instead of PDD, the GD function should be used which has only two values, the first value represents the increasing region and the second one represents the decreasing region of the PDD. So, the GD functions can be used to simplify data representation. Moreover, the GD functions can be applied for easier comparisons of different machines.

The GD function could potentially be helpful for a routine quality assurance program as information of the radiation beam stability over time and general comparisons to other similar machines. Lastly, it is recommended for future studies to use interpolation of the GD function to find other unmeasured energy beam gradients.

\section{References}

1. Khan FM, Gibbons JP. Khan's the Physics of Radiation Therapy. Fifth edition. Lippincott Williams \& Wilkins/Wolters Kluwer; 2014.

2. International Commission on Radiation Units and Measurements, ed. Prescribing, recording, and reporting photon beam therapy (Report 50). Published online 1993.

3. Absorbed Dose Determination in External Beam Radiotherapy. International Atomic Energy Agency; 2001. https://www.iaea.org/publications/5954/absorbed-dose-determination-in-external-beam-radiotherapy

4. Jordan TJ. Megavoltage X-ray beams: 2-50 MV. BJR Suppl. 996;25:62-109.

5. Klein EE, Esthappan J, Li Z. Surface and buildup dose characteristics for 6, 10, and 18 MV photons from an Elekta Precise linear accelerator. J Appl Clin Med Phys. 2003;4(1):1-7. https://doi.org/10.1120/jacmp.v4i1.2537

6. Zhu TC, Palta JR. Electron contamination in 8 and 18 MV photon beams. Med Phys. 1998;25(1):12-19. https://doi.org/10.1118/1.598169 
7. Dogan N, Glasgow GP. Surface and build-up region dosimetry for obliquely incident intensity modulated radiotherapy 6 MV x rays. Med Phys.2003;30(12):3091-3096. https://doi.org/10.1118/1.1625116

8. McKenna MG, Chen XG. Calculation of the dose in the build-up region for high energy photon beam. Treatment planning when beam spoilers are employed. Radiother Oncol. 1995:34(1):63-8. https://doi.org/10.1016/0167-8140(95)01504-a

9. Quach KY, Morales J, Butson MJ, Rosenfeld AB, Metcalfe PE. Measurement of radiotherapy x-ray skin dose on a chest wall phantom. Med Phys. 2000;27(7):1676-1680. https://doi.org/10.1118/1.599035

10. Kim S, Liu CR, Zhu TC, Palta JR. Photon beam skin dose analyses for different clinical setups. Med Phys. 1998;25(6):860-866. https://doi.org/10.1118/1.598261

11. Bilge H, Ozbek N, Okutan M, Cakir A, Acar H. Surface dose and build-up region measurements with wedge filters for 6 and 18 MV photon beams. Jpn J Radiol. 2010;28(2):110-116. https://doi.org/10.1007/s11604-009-0393-5

12. Turner JE. Interaction of ionizing radiation with matter. Health Phys. 2005;88(6):520-44. https://doi.org/10.1097/00004032200506000-00002

13. Xhafa B, Mulaj T, Hodolli G, Nafezi G. Dose Distribution of Photon Beam by Siemens Linear Accelerator. Int J Med Phys Clin Eng Radiat Oncol. 2014;03(01):67-70. https://doi.org/10.4236/ijmpcero.2014.31011

14. Sruti RN, Islam MM, Rana MM, et al. Measurement of Percentage Depth Dose of a Linear Accelerator for 6 MV and 10 MV Photon Energies. Nuclear Sciences and Applications. 2015;24(1):4. 